Teknokultura. Revista de Cultura Digital y Movimientos Sociales

ISSNe: $1549-2230$

http://dx.doi.org/10.5209/TEKN.63113

\title{
Partidos emergentes de la ultraderecha: ¿fake news, fake outsiders? Vox y la web Caso Aislado en las elecciones andaluzas de 2018
}

\author{
Macarena Hernández Conde ${ }^{1}$; Manuel Fernández García ${ }^{2}$
}

Recibido: 31 de enero de 2019 / Aceptado: 13 de marzo de 2019 Open peer reviews

Resumen. El auge actual de nuevos partidos populistas de ultraderecha a nivel global, muchas veces auto posicionados como outsiders o anti-establishment, ha venido acompañado por una preocupación creciente acerca de las estrategias comunicativas que despliegan dichos movimientos, especialmente por su relación con portales de fake news. A raíz de las últimas elecciones autonómicas en Andalucía, con la entrada por primera vez en un parlamento del partido Vox, vuelve a hablarse de la emergencia de una ultraderecha outsider que, ignorada por los medios de comunicación de masas, conquista a su electorado gracias al poder propagador de las redes sociales. ¿Qué función cumplen las webs de fake news en la estrategia de la ultraderecha? ¿se encuentran estas formaciones fuera del espacio de los medios de comunicación de masas? Para responder a estas cuestiones el presente artículo analiza la estrategia comunicativa desplegada por unos de los portales afines a este tipo de partidos durante la campaña electoral andaluza de 2018, con el objetivo de comprobar su nivel de acceso a los medios aplicando los filtros del modelo de propaganda propuesto por Chomsky y Herman. Para ello se realiza un análisis del contenido compartido en redes sociales y un análisis de varias métricas básicas de tráfico a través de herramientas de analítica web. La hipótesis de partida es que la función principal de estos portales no es tanto ejercer como una herramienta para colocar sus temas en la agenda mediática sino reforzar la imagen de outsider de los partidos de ultraderecha.

Palabras clave: agenda; Alt-Right; modelo de propaganda; partidos outsiders; populismo.

\section{[en] Emerging right-wing parties: fake news, fake outsiders? Vox and the "Caso Aislado" website in the 2018 Andalusian elections}

\begin{abstract}
The current rise of new right-wing populist parties at a global level, often self-defined as outsiders or anti-establishment, has received a great amount of attention. Especially, the communicative relations displayed by these movements and their relationship with fake news portals. In the view of what happened in the last elections in Andalusia, with the presence in the parliament of the Vox party, the rise of an outsider right-wing party that, ignored by the mass media, conquers its electorate thanks to the power of social networks is a burning issue. What role do fake news websites play in the strategy of the alt-right? Are these parties outside the agenda of mass media? To answer these questions, this article analyzes the communicative strategy deployed by websites attached to these parties during the Andalusian electoral campaign of 2018, with the objective of establishing their level of access to the media by applying the filters of the propaganda model proposed by Chomsky and Herman. For this purpose, an analysis of shared content in social networks and an analysis of several basic traffic metrics through web analytics tools is carried out. The initial hypothesis is that the main role of these portals is
\end{abstract}

\footnotetext{
$1 \quad$ Universidad de Sevilla (España)

E-mail: machercon@alum.us.es

2 Universidad Pablo de Olavide (España)

E-mail: mfergar1@upo.es
} 
not to be a tool to place their issues on the media agenda but overall to heighten the image of outsiders of the far-right parties.

Keywords: agenda; Alt-Right; outsiders parties; populism; propaganda model.

Sumario. 1. Introducción. 2. Marco contextual: Alt-rigth y fake news. 3. Marco teórico: el modelo de propaganda de Chomsky y Herman en la era de Facebook. 4. Metodología. 5. Caso de estudio: Caso Aislado. 6. Caso Aislado y el modelo de propaganda de Chomsky y Herman. 7. ¿Fuera de la agenda? Conclusiones y cuestiones para el debate. 8. Referencias.

Cómo citar: Hernández Conde, M. y Fernández García, M. (2019). Partidos emergentes de la ultraderecha: ¿fake news, fake outsiders? Vox y la web Caso Aislado en las elecciones andaluzas de 2018 , en Teknokultura. Revista de Cultura Digital y Movimientos Sociales, 16(1), 33-53.

\section{Introducción}

El auge de la ultraderecha, o del populismo autoritario (Hall, 2018), como el representado por Donald Trump en EE. UU., Jair Bolsonaro en Brasil o Matteo Salvini en Italia han venido acompañados de una creciente atención en lo que respecta a la transformación de sus estrategias comunicativas. Términos como posverdad o fake news se repiten a diario en los análisis del éxito de estas formaciones que, según su propio discurso, han crecido pese a la marginación de los medios de comunicación tradicionales.

Tras las últimas elecciones autonómicas andaluzas, con el inesperado éxito de Vox (12 diputados frente a los 0-3 que les daban las encuestas) vuelve a hablarse, en este caso en nuestro país, de la emergencia de una ultraderecha outsider que, ignorada por los medios de comunicación de masas, conquista a su electorado gracias al poder propagador de redes sociales como Facebook o WhatsApp.

En este sentido, se han multiplicado la creación de páginas webs y perfiles en redes sociales que propagan contenidos afines a la estrategia de estas formaciones $y$, en muchas ocasiones, falsos. En relación con esto, el presente artículo se plantea las siguientes preguntas ¿qué función cumplen las webs de fake news en la estrategia de comunicación de formaciones emergentes de ultraderecha? ¿se encuentran estas formaciones fuera del espacio de los medios de comunicación de masas?

La hipótesis de partida es que la función principal de estos portales no es tanto ejercer como una herramienta para colocar sus temas en la agenda mediática puesto que estos temas ya están presentes, sino reforzar la imagen de outsider de los partidos de ultraderecha, concretamente de VOX.

\section{Marco contextual: Alt-rigth y fake news}

Donald Trump pareció abrir la brecha de una nueva derecha populista que asegura no contar con el apoyo del establishment ni de los medios de comunicación tradicionales, ganando las elecciones en noviembre de 2016 y convirtiéndose en presidente sin el apoyo expreso de su partido. Pronto otros los líderes políticos del populismo de 
derechas como Matteo Salvini en Italia y Jair Bolsonaro en Brasil siguieron su estela, haciendo uso intensivo de las redes sociales frente a los medios de comunicación tradicionales para llegar a sus electores y alcanzar la victoria.

Entendemos que esta nueva ultraderecha se engloba en lo que Stuart Hall definió como populismo autoritario: "una forma excepcional del Estado capitalista que, a diferencia del fascismo clásico, ha mantenido en su lugar a la mayoría (aunque no todas) las instituciones formales de representación y, a la vez ha sido capaz de concitar en torno a él un consentimiento popular activo" (Hall, 2018, pp. 82-83). Analizando estos casos, se observan algunas características comunes. En la campaña de Donald Trump, ¿se puede afirmar que los medios de comunicación de masas hicieron un frente común contra su campaña? Trump se sirvió de Twitter para sortear a la prensa tradicional y hablar a sus electores directamente, aprovechando además para deslegitimar a los medios de comunicación críticos acusándolos de tendenciosidad y de propagar noticias falsas (Vlatković, 2018). Donald Trump es un personaje famoso y millonario, bien posicionado en los medios online y offline (Pérez Curiel y Limón Naharro, 2019). Además, su posición como elemento incómodo para los medios de comunicación es aún más cuestionable si tenemos en cuenta que fue apoyado por la poderosa cadena de noticias estadounidense Fox, que pertenece a News Corp propiedad del magnate Rupert Murdoch, que posee entre otros medios: New York Post, The Wall Street Journal, Sky News o la editorial Harper Collins. La estrategia de Murdoch ha sido estudiada y definida como:

Un altavoz mediático conservador particularmente persistente. Los intereses empresariales y táctico-políticos coexisten en la experiencia estadounidense de News Corporation con motivaciones ideológicas. Murdoch ha contribuido a que el aislamiento mediático del movimiento conservador estadounidense sea un hecho del pasado. Lo que fue un hueco de mercado aprovechado por el magnate ahora se ha generalizado; la inteligencia de Murdoch ha consistido en anticiparse (salvo excepciones) a todo ello, haciendo converger intereses empresariales y convicciones ideológicas (Pineda y Romero, 2011, pp. 210-211).

Sobre la coincidencia de intereses económicos e ideológicos volveremos más adelante. Por otro lado, al recientemente electo presidente de Brasil, Jair Bolsonaro, se le achaca haber ganado las elecciones gracias a una campaña masiva de difusión de 'fake news' a través de la aplicación WhatsApp. El diario Fohla de S. Paolo reveló tras una investigación que un grupo de empresarios habría invertido al menos $12 \mathrm{mi}$ llones de reales (2,8 millones de euros) para comprar paquetes de mensajes masivos de WhatsApp con contenido denigrante para su principal rival en las elecciones, el izquierdista Partido de los Trabajadores (Campos Mello, 2018). Por tanto, tampoco la campaña de Bolsonaro puede considerarse, pues, ajena a los intereses de ciertas elites económicas.

\section{1. ¿Qué hay de nuevo en las fake news?}

En los últimos tiempos, existe una creciente preocupación acerca de la proliferación de noticias falsas que pretenden influenciar los procesos políticos, especialmente los de ámbito electoral. Así, a raíz de las acusaciones al gobierno ruso de utilizar las re- 
des sociales como herramienta de desinformación, la Comisión Europea ha creado el grupo de trabajo East StratCom y un código de Buenas Prácticas contra las fake news (European Commission, 2019), definiendo estas como "una simplificación del complejo problema de la desinformación. La desinformación puede ser entendida como información falsa creada deliberadamente y difundida para influenciar la opinión pública o enmascarar la verdad. La desinformación no incluye los errores periodísticos, la sátira o la parodia" (European Commission, 2019).

Realmente, las noticias falsas no representan una novedad, si bien anteriormente se hablaba preferentemente de propaganda de guerra o desinformación. Lo que sí supone una novedad es la velocidad con la que estas son difundidas y el alcance que logran. Esto es debido principalmente a la expansión de Internet y al uso intensivo de las redes sociales. Desde la teoría contemporánea se definen las fake news como "publicaciones virales basadas en historias ficticias que parezcan noticias" (Tandoc et al., 2018, p. 138).

Las motivaciones de las fake news pueden ser principalmente económicas o ideológicas. Tienden a ser sensacionalistas y provocadoras, destinadas a conseguir un mayor impacto y, por consiguiente, más ventas. Es lo que hoy en día, en el ecosistema mediático digital, se traduce por maximizar el número de personas que cliquee en una noticia debido al titular llamativo, lo que se conoce como "clickbait", generando así más ingresos publicitarios. El uso de las fake news con motivos ideológicos (aunque también económicos) está bien documentado, por ejemplo, en la Guerra de Irak, con la noticia publicada por una periodista del New York Times dando por buena la existencia de armas de destrucción masiva y que justificó, en última instancia, la posterior invasión (BoydBarrett, 2004).

El impacto del uso de fake news en campañas electorales ha sido estudiado recientemente por diversos autores en Estados Unidos (Bovet y Makse, 2019; Knight Foundation, 2018). Estos estudios se centran en la influencia que puedan tener sobre el proceso político y, si bien ha quedado establecida la exposición de una amplia mayoría de la población a las fake news, no puede comprobarse su influencia real en el voto de la ciudadanía (Allcott y Gentzkow, 2017). Además, el consumo de fake news está altamente concentrado en pequeños grupos y, aunque 1 de cada 4 estadounidenses visitó webs de fake news durante las elecciones de 2016, 6 de cada 10 visitas provenían de un $10 \%$ de estos usuarios (European Research Council, 2018).

Es relevante, sin embargo, su capacidad para fijar temas, tendencias y posiciones en el debate público. Es necesario tener en cuenta que, en España, según la encuesta del Centro de Investigaciones Sociológicas (CIS, 2017), el 67'8\% de la población accede a Internet con asiduidad y de estos el $40,2 \%$ se informa sobre campañas electorales a través de la web de medios de comunicación, un $25,1 \%$ a través de redes sociales y un $6,2 \%$ a través de otro tipo de páginas web. Según esta misma encuesta, el 50,8\% sigue la información política a diario a través de la televisión, un 19,8\% a través de los periódicos y el 17,5\% a través de la radio. Por lo que la relevancia de los discursos implantados en los medios de comunicación de masas, tanto en su versión online como analógica, siguen siendo la principal fuente de información política actual. Esto aporta una panorámica de la influencia que las fake news pueden tener en el proceso político español.

\subsection{La alianza de la alt-right}

La extrema derecha de EE.UU. (conocida como Alternative Right o alt-right) ha marcado tendencia con una nueva estrategia comunicativa más agresiva, que apro- 
vecha la cultura de internet y el discurso de la contracultura. Uno de sus principales ideólogos fue el director de la campaña que llevó a Trump a la victoria, Steve Bannon. Bannon era responsable de la web Breitbart News, una plataforma de noticias de periodistas y escritores vinculados a la ultraderecha que alcanzó popularidad durante las elecciones de 2016 publicando conspiraciones y noticias falsas sobre Hillary Clinton (Papetti, 2016). Se le considera, asimismo, responsable del 'algoritmo populista' que ideó con la colaboración de Cambridge Analytica, la empresa de minería de datos contratada durante la campaña de las elecciones de 2016, que obtuvo millones de datos de usuarios de Facebook bajo la excusa de estudios académicos para realizar una microsegmentación de los mensajes lanzados en la campaña de Donald Trump (Cadwalladr y Graham-Harrison, 2018). Una de sus principales líneas discursivas es el mensaje anti-establishment y el posicionamiento como outsider con un mensaje antiinmigración, patriótico, antifeminista e islamófobo (Pérez Curiel y Limón Naharro, 2019; Rachman y Yunianti, 2017).

Eduardo Bolsonaro, hijo del nuevo presidente de Brasil, Jair Bolsonaro, y diputado federal de Brasil, mantuvo un encuentro con Bannon y anunció en sus redes sociales que sería un consejero ocasional en la campaña de su padre. Steve Bannon también ha sido vinculado con otros grupos de ultraderecha en Europa a través del think tank "The Movement" que ha conseguido interesar a partidos de ultraderecha de Bélgica, Italia, Francia o España. Su vinculación con Vox ha sido reconocida uno de sus dirigentes, Rafael Barjadí, ex asesor del expresidente español José María Aznar y vinculado a la fundación que este dirige, FAES. En una entrevista tras un encuentro entre ambos afirmó: "Me dijo que estaba pensando en montar un think tank en Europa para coordinar mensajes y que Vox tenía que participar de alguna manera. Nos ofreció su aparato tecnológico para movernos en las redes sociales con los mensajes adecuados, probar ideas y hacer una campaña electoral al estilo americano" (Junquera, 2018).

\section{Marco teórico: el modelo de propaganda de Chomsky y Herman en la era de Facebook}

En el sistema neoliberal, la prensa y los medios de comunicación de masas no parecen defender el propósito social de ser los garantes del derecho a la información de los ciudadanos para controlar y participar en la esfera política sino más bien tratan de "inculcar y defender el orden del día económico, social y político de los grupos privilegiados que dominan el Estado" (Chomsky y Herman, 2009, p. 341). Es decir, son empresas que, entre otras, cumplen una función propagandística al servicio de las élites. Para ello, conforman una agenda mediática que incluye los temas que serán ofrecidos a la opinión pública y cómo serán tratados, así como aquellos que serán silenciados. Este es el punto de partida del modelo de propaganda que proponen Chomsky y Herman (2009). Estos autores establecen, además, una serie de filtros que consiguen que ciertos temas sean capaces de introducirse en la agenda mediática y aquellos que sean opuestos a los intereses de las élites se vean silenciados.

Chomsky y Herman desarrollan por primera vez su modelo en 1988 con Los guardianes de la libertad y aunque lo retoman posteriormente en otras obras (Chomsky, 2007), hemos creído necesario partir de las actualizaciones del modelo que han desarrollado Sierra y Vázquez (Sierra y Vázquez, 2006). 
En primer lugar, es necesario asumir que los medios no se limitan a transmitir información, sino que construyen la realidad, abandonando el pretendido paradigma de la objetividad. Es de especial relevancia el principio enunciado como simbiótico de la hegemonía en tanto se puede establecer que los discursos que subyacen en las fake news pese a su carácter pretendidamente extremista se engarzan dentro de una red de discursos estratégicos convergiendo en la reproducción social de un discurso hegemónico (Sierra y Vázquez, 2006).

Por otra parte, también son de interés las aportaciones hechas al modelo por Joan Pedro, sobre todo, las pertinentes al quinto filtro sobre el anticomunismo y la adición de la tecnología como filtro a contemplar (Pedro, 2009).

\subsection{Primer filtro: la propiedad. Internet, ¿un espacio para la democratización de la comunicación?}

El paisaje dibujado por Herman y Chomsky (Chomsky y Herman, 2009) de unos medios de comunicación cada vez en manos de menos empresas no ha hecho sino acrecentarse. Las industrias culturales actuales están concentradas en megacorporaciones transnacionales (McChesney, 2008). La diversificación sectorial de estos conglomerados lleva emparejada una confrontación de intereses empresariales que, necesariamente, influirán en la línea informativa de los medios asociados al grupo.

La llegada de Internet y los medios de comunicación online han supuesto un cambio en el concepto de propiedad de los medios. Si bien los costes de un producto digital se reducen considerablemente, para poder competir con el alcance de los medios tradicionales debemos conseguir visibilidad. ¿Es Internet, por tanto, una posibilidad para la democratización de la comunicación?

Frente a las posturas tecno-optimistas que defendían Internet como una herramienta democratizadora capaz de subvertir el control para definir la agenda mediática, se dibuja un panorama marcado por la presencia de megacorporaciones que cada vez ejercen más control. La amenaza a la neutralidad de la red y el perfilado y control sobre nuestros datos dibujan un escenario poco halagüeño, como afirma McChesney: "La tremenda promesa de la revolución digital se ha visto comprometida por la apropiación y el desarrollo capitalista de Internet" (McChesney, 2013, p. 97). El uso de las redes sociales como herramienta capaz de saltarse los filtros que impiden el acceso a la agenda mediática se encuentra con varios obstáculos. En primer lugar, la propiedad de estas redes sociales que, al igual que los medios de comunicación de masas, están en manos de grandes corporaciones (McChesney, 2013).

En segundo lugar, la progresiva priorización de los beneficios por encima de la función social en las empresas informativas y la creciente precarización de las condiciones de trabajo (McChesney, 2008) suponen una bajada en la calidad informativa y una exigencia para conseguir contenidos 'populares'. Este aspecto tendría relevancia dentro de uno de los filtros propuesto por Joan Pedro (Pedro, 2009), la tecnología como condicionante de la naturaleza y forma de las noticias. Si trasladamos esta lógica del proceso comunicativo a las redes, se detectan tres factores de la lógica de la red que están influyendo en la comunicación política: una producción individual sin entornos profesionales que fijen lo que es noticiable, la distribución marcada por la lógica de la viralidad más que la relevancia o responsabilidad social y la recepción marcada por el efecto burbuja y sesgo de confirmación (Klinger y Svensson, 2014). 
Estas tendencias abren preguntas sobre si las redes sociales están favoreciendo una suerte de 'comunicación populista' ampliada y reflejada en los medios ya que suponen un entorno propicio donde se da una suerte de "'actividad de 'remix', un collage creativo de clips de video, piezas de sonido, clickbaits, graffiti, parodias, memes y otros muchos contenidos, incluyendo insultos y fake news, que se muestran cruciales para incrementar la popularidad del líder, de su sistema de creencias, de sus bases" (Mazzoleni y Bracciale, 2018).

\subsection{Segundo filtro: publicidad y modelo de negocio}

La forma de consumir a través de Internet ha supuesto un cambio en la economía de los medios de comunicación y sus formas de producir. La consagración de las redes sociales ha generado una disminución en los ingresos de la prensa y esto ha supuesto la lógica del clickbait en los medios digitales (o en las versiones digitales de los medios tradicionales) que ha cambiado el lenguaje de la prensa. Los titulares llamativos que incitan a cliquear en el enlace para leer más y saber qué ha ocurrido son la norma, el cebo del "clickbait".

Como veíamos en el caso de la cadena de noticias conservadora Fox News (Pineda y Romero, 2011), no se trata sólo de ideología, detrás hay un rentable modelo de negocio. La publicidad automática por Internet recompensa un tipo de contenido. Las webs participan en plataformas de publicidad automática de Google y otras compañías. Se trata de un modelo de publicidad en la que se compran audiencias y no espacios, de modo que un algoritmo coloca el anuncio en la web indicada cuando el perfil del visitante de la web coincide con el target de la marca. Cuantos más visitantes lleguen a la web y vean los anuncios, mayores serán los beneficios. Este tipo de publicidad, conocida como programática, es un negocio para Google, que percibe una parte de los ingresos.

\subsection{Tercer filtro: dependencia de las fuentes}

Las fuentes a las que se tenga acceso marcaran los contenidos disponibles para elaborar las noticias. Entendemos que los partidos políticos pueden subvencionar en cierto modo a los medios cercanos proporcionándoles materiales ya elaborados, así como acceso privilegiado a la información y, por supuesto, con la compra de espacios publicitarios ya sea directamente o a través de las instituciones públicas que controlan.

La precarización de la profesión y la velocidad de producción de contenidos que impone el ecosistema digital son factores que impulsan a los medios a hacerse más dependientes de las informaciones ya elaboradas desde partidos e instituciones y a descartar la búsqueda de otras fuentes o el contraste de los datos.

\subsection{Cuarto filtro: los reforzadores de opinión}

La presión ejercida por grupos de interés sobre los medios de comunicación es otro de los factores que puede influir y controlar los contenidos presentes en los medios de comunicación. Estos grupos de interés pueden están respaldados por corporaciones o intereses económicos que implican que a los medios les resulte perjudicial económicamente defender su posición. Por ejemplo, ante la denuncia sistemática de un 
programa por parte de una organización o un sector de la ciudadanía, los anunciantes publicitarios acaban por rehuirlo para evitar publicidad negativa y pueden conseguir que el programa sea retirado.

\subsection{Quinto filtro: anticomunismo y convergencia en la ideología dominante}

El comunismo ataca la base de la élite económica y del poder: la propiedad privada. Aunque parezca que este filtro ha perdido actualidad desde la desintegración de la Unión Soviética, su importancia sigue vigente: "En los países occidentales, toda referencia a políticas alternativas en el ámbito económico es descalificada y representada marginalmente por el discurso anticomunista reinante en los medios" (Sierra y Vázquez, 2006, p18). Obliga a los liberales a colocarse en una posición defensiva y supone un acoso a los socialdemócratas si no demuestran suficiente rechazo a los 'radicales', ejerciendo presión para demostrar sus credenciales anticomunistas.

Aun así, preferimos la revisión que de este filtro realiza Joan Pedro al hablar de Convergencia en la Ideología Dominante, que supone aquella que "está instaurada en el seno del periodismo, lo que proporciona una base moral, ideológica, normativa y de actuación que hace que la propaganda a favor de "nuestro bando" (definido por las élites) parezca legítima y necesaria” (Pedro, 2009).

Aunque originado en el análisis de EE.UU., el modelo de propaganda sigue vigente como herramienta de análisis, no solo en ese contexto, sino también en otros países, ya que cada vez más, la concentración de medios es un fenómeno global. Así, el modelo de propaganda desarrollado por Chomsky y Herman ha sido empleado en estudios en diferentes contextos como puede ser el caso de la construcción de la imagen sobre Cuba en la prensa norteamericana (Gómez Masjuán, 2010) o el tratamiento de los atentados del 11-S en España (Camiñas Hernández, 2003).

El modelo propuesto por Chomsky y Hermann puede ser útil para establecer la existencia de un sistema de propaganda auspiciado por las élites corporativas y gubernamentales y, por tanto, para ayudar a esclarecer los mecanismos que se ponen en marcha para permitir que determinados discursos se incorporen a la agenda mediática y otros no. Debido, además, a las profundas transformaciones que han sufrido los medios de comunicación en esta época (McChesney, 2008, 2013) es posible afirmar que el modelo está más vigente que nunca y las aportaciones de Sierra y Vázquez (2006) permiten contextualizarlo dentro de la construcción de un consenso envolvente que busca establecer su hegemonía por medio de múltiples dispositivos para apuntalar esa ideología dominante.

\section{Metodología}

Estableceremos como caso de estudio la web Caso Aislado, un portal vinculado a Vox que ha sido denunciado en varias ocasiones por la construcción de bulos y noticias falsas.

Realizaremos un análisis descriptivo del objeto de estudio y analizaremos varias métricas básicas de tráfico web a través de la herramienta online de ana- 
lítica web Similarweb ${ }^{3}$. Una vez establecida Facebook como la principal fuente de tráfico para nuestro caso, usamos la aplicación Netvizz ${ }^{4}$ (Rieder, 2013) que nos permite extraer y visualizar una serie de datos de Facebook. Para el presente estudio se analizan los posts publicados en el rango de fechas seleccionado. Se ha optado por analizar los posts compartidos por Caso Aislado durante la campaña electoral andaluza (del 15 al 30 de noviembre, ambos inclusive), al ser un periodo de intensa actividad para las formaciones políticas y en la que Vox despegó con fuerza. Se analizarán el eje temático de la noticia compartida y las reacciones a dichas noticias (número de likes y número de veces que se ha compartido).

Una vez obtenidos los datos, se realizará un análisis de contenido. Como unidad de análisis se partirá de los titulares de las noticias, metodología empleada en numerosas investigaciones (Andreu, 2008). En una primera revisión de las noticias se confeccionará una lista de categorías en las que se engloban las noticias de modo inductivo. Siguiendo la metodología propuesta por Andreu (Andreu, 2012) para decodificar la agenda mediática, se ha tenido en cuenta los ítems obtenidos a partir del barómetro del CIS del mes de diciembre de 2018 (CIS, 2018) sobre los principales temas de preocupación de la ciudadanía a la hora de realizar un primer nivel de análisis que pueda guiarnos en nuestra categorización. Para ello se ha realizado un análisis textual cuantitativo de los titulares buscando los focos temáticos y sus principales atributos. Tras un análisis inductivo, se han obtenido las siguientes categorías presentes en las noticias compartidas en la página de Caso Aislado en el periodo analizado:

- Cataluña

- Feminismo (problemas de la mujer)

- Inmigración:

- ayudas públicas,

- delitos cometidos por inmigrantes,

○ islam;

- Patriotismo,

- Ataques a Vox de:

- medios de comunicación,

$\circ$ otros ataques o agresiones.

Por otro lado, analizaremos la presencia de los diferentes actores políticos junto con tratamiento que se les da en la noticia (positivo, negativo o neutro). En último lugar, se aplicará cada uno de los filtros del modelo de propaganda de Chomsky y Herman a nuestro caso de estudio con el objetivo de comprobar si estos temas pueden tener cabida en la agenda mediática.

3 SimilarWeb (https://www.similarweb.com) es un sitio web que proporciona servicios de análisis web para empresas. La compañía ofrece a sus clientes información sobre los volúmenes de tráfico de los sitios webs, fuentes de referencia, incluyendo análisis de palabras clave; y "stickiness" del sitio web, entre otras características.

$4 \quad$ Netvizz es una aplicación de Facebook desarrollada por Bernhard Rieder (profesor de la Universidad de Ámsterdam) que te permite crear gráficos a partir de los datos extraídos de una página o perfil de Facebook. 


\section{Caso de estudio: Caso Aislado}

Caso Aislado ${ }^{5}$ es un portal web de noticias que ha sido definido por Maldita.es ${ }^{6 c}$ omo uno de los mayores creadores de fake news. Se creó en 2016 y el resto de proyectos en los que ha participado su creador son portales que buscan un crecimiento rápido de visitas y generar beneficios por publicidad como intergoles.com o el Grupo Hoy.

Como autor habitual de la sección de opinión encontramos al candidato número 8 de Vox en Málaga, David Vasegh Castro, y coordinador de Vox Fuengirola. También fue colaboradora habitual, antes de fichar por el PP, Yolanda Couceiro, militante del Partido por la Libertad, formación de extrema derecha, con el rechazo a la inmigración y al islam como bandera y actualmente en conversaciones con Vox para integrarse.

La publicidad insertada en su página también muestra una clara cercanía ideológica con dicha formación: como publicidad directamente insertada en su web, encontramos banners del programa Alt News de Cadena Ibérica, una radio online "volcada en la recuperación de la historia de España, en el análisis político y en la transmisión de valores culturales y religiosos" según los metatags de su web, o la Editorial Kalma que recientemente ha publicado el libro "La España Viva. Conversaciones con 12 dirigentes de VOX".

Es sobre todo en los ejes temáticos de las noticias publicadas donde, como veremos posteriormente, hay mayor coincidencia con los ejes temáticos de la formación ultraderechista. Según las herramientas de análisis web en diciembre de 2018, Caso Aislado obtuvo 1.000.000 visitas únicas. El 47\% de su tráfico procede de las redes sociales, por lo que la viralidad de sus contenidos es un aspecto esencial. Nos parece interesante iniciar un esbozo de la existencia de un ecosistema de webs de ideología ultraderechista que comparten contenidos.

Figura 1. Procedencia y destino de las visitas de la web de Caso Aislado (octubre-diciembre 2018). Fuente: elaboración propia con Similarweb

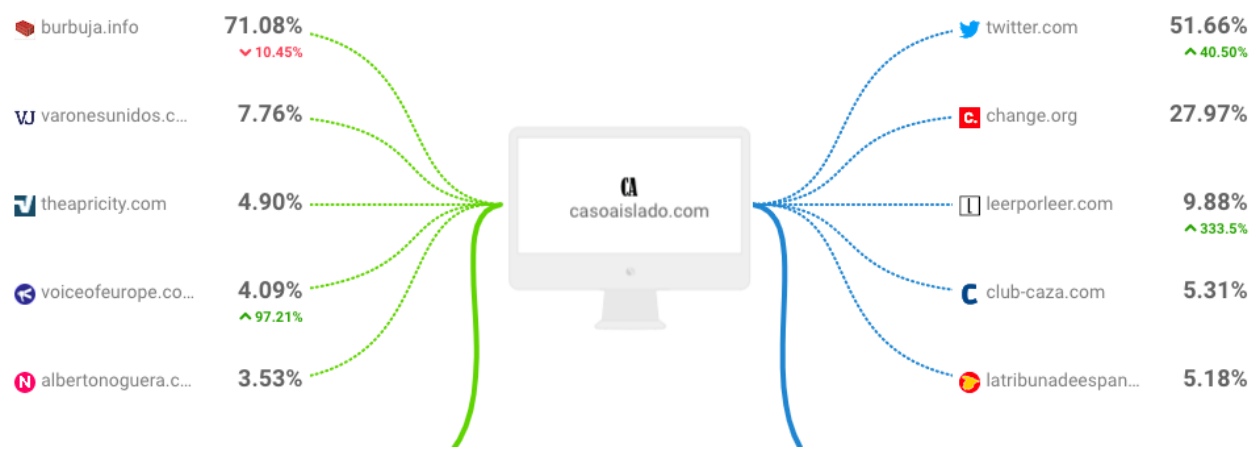

Las menciones provienen en su mayor parte de burbuja.info, un foro sobre economía en cuyo subforo de política, Vox tiene gran protagonismo. En segundo lugar se

\footnotetext{
5 https://casoaislado.com/
} 
encuentra la web varonesunidos.com un portal de noticias falsas contra el feminismo y en menor medida en el subforo español de The apricity, una comunidad entorno a la cultura europea o la web de noticias Voice of Europe, con un discurso antiinmigración.

Desde la web de Caso Aislado se enlaza a otras webs como La Tribuna de España, que se define como "el arma mediática contra el pensamiento único. Periodismo cristiano y patriota, azote de la corrupción e insumiso con los poderosos", o la web de recomendación de libros Leer por leer que destaca el anteriormente mencionado "La España Viva. Conversaciones con 12 dirigentes de VOX".

En el siguiente gráfico (figura 2) es posible observar el solapamiento de audiencias con otros medios y webs digitales. Es decir, qué otras webs visitan los usuarios de Caso Aislado, pudiendo conformarse un ecosistema de medios digitales divididos en dos esferas. Por un lado un sector donde priman las webs de tendencia ideológica de derechas (Okdiario, cuyo fundador y director es el tertuliano Eduardo Inda, Mediterráneo Digital, esdiario.com, fundada por el ex dirigente de Alianza Popular Antonio Martín Beaumont, Periodista Digital, del periodista adscrito a círculos ultraderechistas Alfonso Rojo, y Libertad Digital, fundado por el periodista Federico Jiménez Losantos que ha manifestado su cercanía a Vox (Jiménez Losantos, 2018) pero donde se mezclan también webs de fuentes oficiales (congreso.es), medios tradicionales en su versión digital (diariodecadiz.es, larazon.es) o webs que generan noticias falsas de modo sarcástico (eljueves.es, elmundotoday.com).

Figura 2. Solapamiento de la audiencia de Caso Aislado con otras webs (octubre-diciembre 2018). Fuente: elaboración propia con Similarweb.

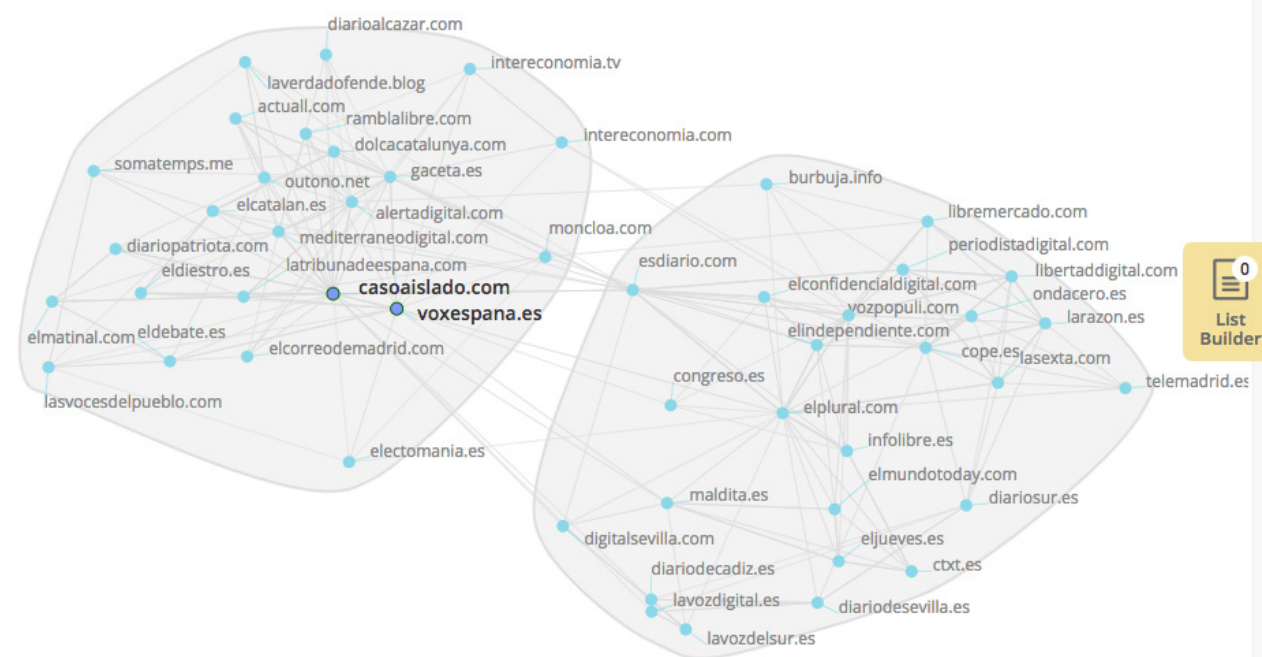

Las principales redes sociales donde las noticias de la web son compartidas (y de dónde proviene el tráfico) son Facebook, de donde proceden el 65,67\% de las visitas y Twitter, de donde proceden el 33,7\%. 
Figura 3. De dónde proceden las visitas a la web (octubre-diciembre 2018).

Fuente: elaboración propia con Similarweb.

\section{Social Traffic}

Oct 2018 - Dec 2018, $\odot$ Worldwide 9 Desktop Only
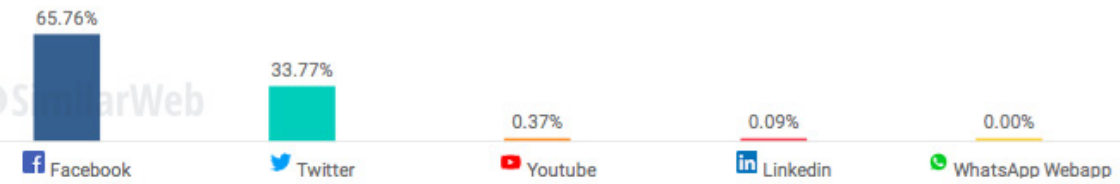

\subsection{Análisis de contenido}

Dado que Facebook es la primera fuente de viralización de noticias, hemos seleccionado las noticias compartidas en esta red durante el periodo de la campaña electoral andaluza. Hemos analizado los posts a dos niveles: en cuanto a ejes temáticos y presencia y valoración de diferentes formaciones políticas.

Figura 4. Categorías temáticas de las noticias compartidas por Caso Aislado en Facebook (15-30 de nov 2018). N=50. Fuente: elaboración propia a partir de datos de Facebook.

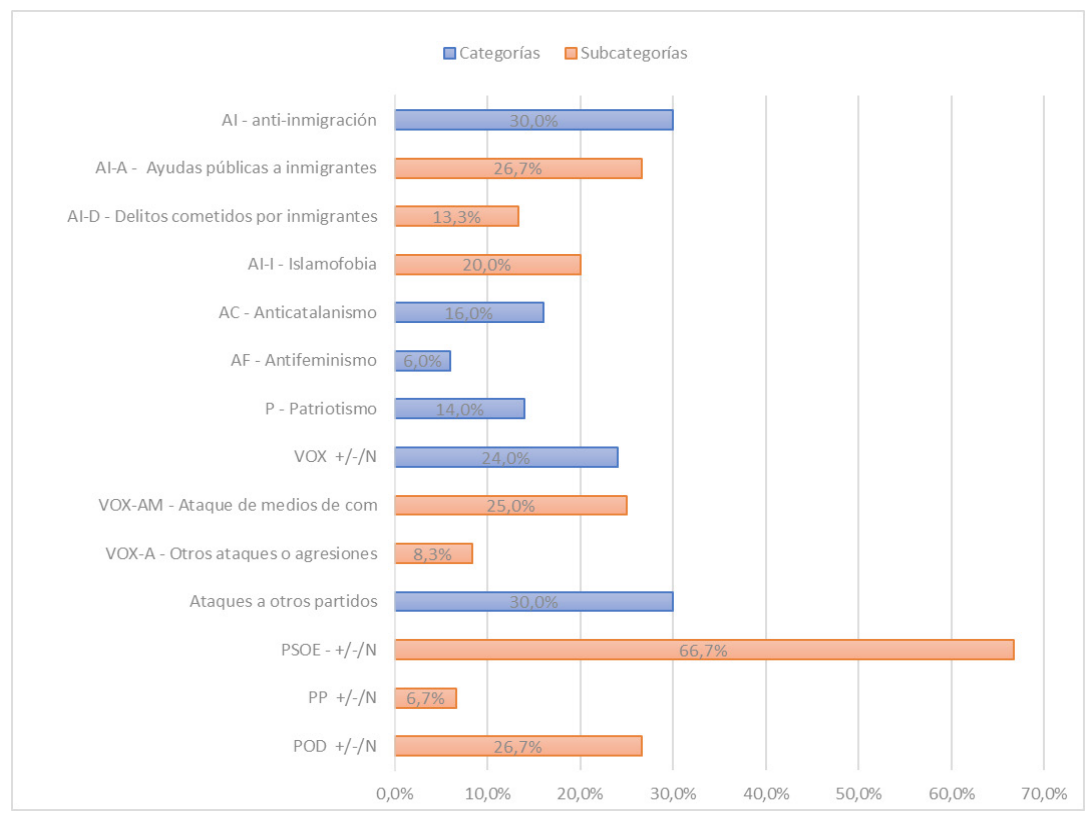

Como se observa en la figura 4 el $30 \%$ de las noticias publicadas responden a noticias contra la inmigración, de ellas el $26^{\prime} 6 \%$ vinculan a inmigrantes con la comi- 
sión de delitos, el $20 \%$ son contenidos de carácter islamófobo y el 13\%3\% anuncian diferentes ayudas y recursos públicos supuestamente recibidas por inmigrantes. El segundo tema más relevante son noticias anticatalanistas que ascienden al 16\% del contenido publicado. Le siguen con un $14 \%$ noticias de contenido patriótico, fundamentalmente en defensa de los símbolos y la bandera española. Por último, con un $6 \%$ encontramos noticias contra el feminismo.

En cuanto a la presencia y valoración de formaciones políticas, VOX es protagonista del $24 \%$ de las noticias compartidas en la red social, con dos líneas muy marcadas, un $66^{\prime} 6 \%$ de las noticias que protagoniza son noticias valorando positivamente acciones y medidas promovidas por la formación y el $25 \%$ recogen presuntos ataques de medios de comunicación a esta formación, reforzando el supuesto papel marginal al que son sometidos por los medios de comunicación de masas. Un 8’3\% son ataques contra la formación cometidos por otros personajes públicos. Por otra parte, las menciones al resto de partidos del espectro político son siempre en clave negativa, entre estos se repiten los ataques al PSOE incluyendo en estos los dirigidos contra el presidente del gobierno o la presidenta de la Junta de Andalucía, a Podemos $\mathrm{y}$, en menor medida, al Partido Popular.

De media, las noticias publicadas obtuvieron 192 reacciones y fueron compartidas una media de 298 veces, con un total de 14.932 compartidos a lo largo de la campaña electoral. Si atendemos al volumen de compartidos, Caso Aislado adquiere un lugar relevante dentro de los medios nativos digitales frente a los, por ejemplo, 9.981 compartidos que obtuvo Público en el mismo periodo de tiempo o los 12.725 compartidos de eldiario.es.

Siendo conscientes de la limitación del análisis realizado, es posible afirmar que los ejes discursivos detectados y la presencia y tratamiento positivo de Vox en las noticias, permiten establecer una cercanía ideológica y afinidad con el discurso entre dicha formación y la web analizada. Así, en las categorías discursivas principales de Vox ocupa un lugar destacado la afirmación patriótica, el control de la inmigración y las fronteras y la lucha contra los principios feministas (Abascal, 2018). Por otro lado, el tratamiento victimista de Vox en relación a otros partidos refuerza su posicionamiento como fuerza marginada y perseguida por los medios de comunicación tradicionales.

\subsection{Posicionamiento outsider}

Entendemos la figura del outsider no sólo de manera general como el que "viene de fuera" sino, aplicado a la política, como el que viene de fuera de la política tradicional y de las élites, siendo esto entendido como un valor en alza ante la creciente desafección ciudadana (Rodríguez Andrés, 2016). El análisis de contenido nos marca una clara dirección del discurso de la formación política Vox, colocándose como víctimas atacadas por los medios de comunicación de masas. Esta representación se extiende a los medios de comunicación digitales que hemos vinculado a esta formación, en primer lugar, la propia Caso Aislado, cuyos redactores se presentan como periodistas incómodos para el sistema: "el director de CasoAislado.com. Tiene unas ganas tremendas de contar la verdad que otros tratan de ocultar. Las críticas le hacen más fuerte", "Apasionada del periodismo. Sin miedo a escribir de temas polémicos. Me criticarán por lo que escribo, pero la verdad a veces duele". 
El resto de la prensa digital que comparte audiencia con esta web sigue la misma línea discursiva: el lema de Okdiario es "el sitio de los inconformistas", mientras que el de Mediterráneo Digital es "políticamente incorrecto". La propia Caso Aislado se describe en su página de Facebook con la siguiente afirmación: "Si lo que publicamos molesta, es porque algo estamos haciendo bien. Medio de comunicación independiente. Hablamos de lo que otros medios se niegan a hablar".

Centrándonos en el caso de estudio, nos parece relevante el estudio comparativo realizado entre la cobertura recibida por Vox y la obtenida por PACMA (Partido Animalista contra el Maltrato Animal) que obtuvo más votos en las anteriores generales y andaluzas. El estudio demuestra que la cobertura a Vox era bastante superior incluso cuando las intenciones de voto a PACMA eran considerablemente superiores. Cuando la intención de voto de Vox se incrementó, su cobertura aumentó aún más. También se destaca que la cobertura de Vox se asemejó más a la de los grandes partidos que contaban con mucha más intención de voto y presencia previa en las instituciones (Olalla et al., 2019).

Figura 6. Cobertura en El Mundo y El País en las elecciones andaluzas de 2018.

Fuente: Olalla et al., 2019.

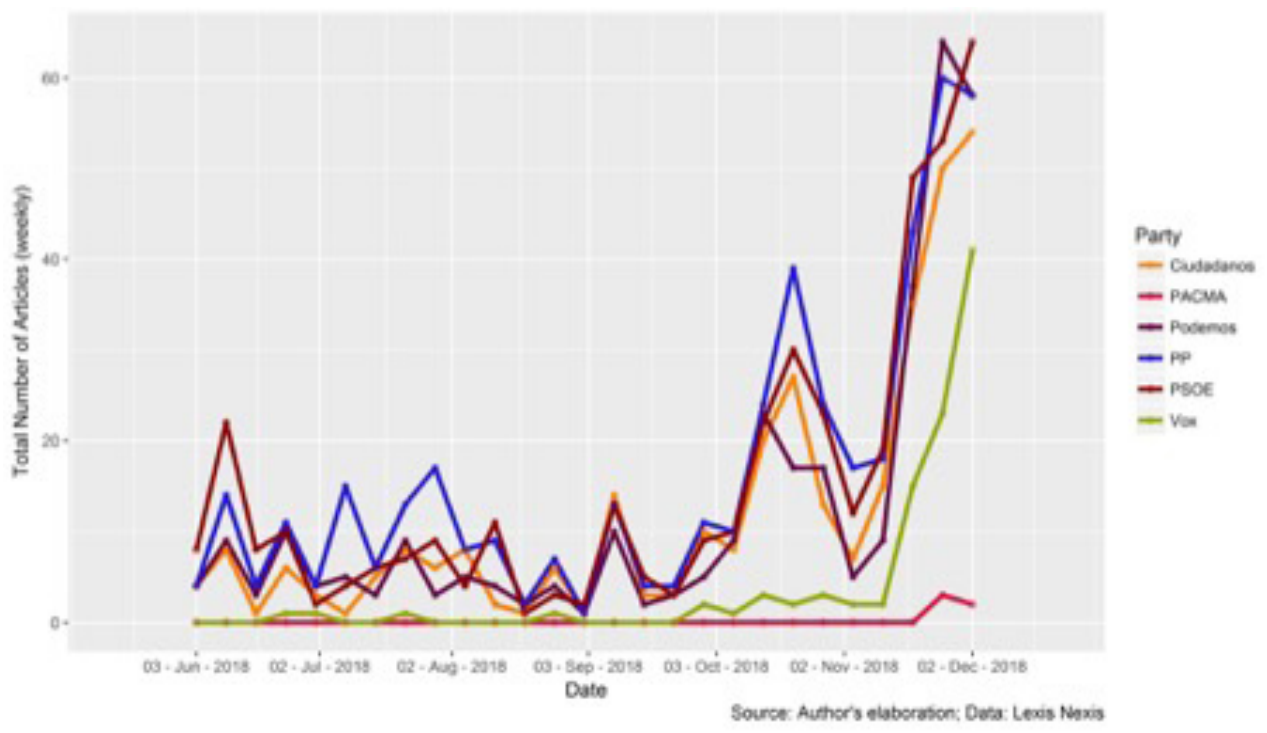

\section{Caso Aislado y el modelo de propaganda de Chomsky y Herman}

\subsection{Primer filtro}

La caída de la publicidad y la crisis de los medios de comunicación tradicionales unidas a la aparición de nuevos medios digitales crea un panorama mediático am- 
pliamente competitivo donde la estrategia de Caso Aislado de conseguir viralidad, clics y visitas como objetivo prioritario, marca el contenido de la página.

Caso Aislado nutre a las redes sociales de contenido de impacto y se alimenta del tráfico que la viralidad le reporta. Desde el discurso de Vox se hace una falsa equiparación, el hecho de usar una plataforma independiente se anuncia como la única manera de acceder al debate público ya que no se tiene presuntamente acceso a los medios de comunicación tradicionales que recogen el marco de opinión de las élites simbólicas. El hecho de acceder a la visibilidad gracias a la viralidad (a la decisión de la gente) les permite venderse como contrahegemónicos frente al discurso dominante y así lo anuncia Manuel Mariscal, responsable de redes de Vox, en los propios medios a los que se supone que no tienen acceso: «No necesitamos a los grandes medios. Te dan prestigio, pero para llegar a ese público que nos interesa y que vive pegado al móvil nos basta con las redes. En EEUU Trump ganó sin el apoyo de los medios tradicionales. Y tenemos futuro porque no dependemos de ellos" (Negre, 2018).

\subsection{Segundo filtro: publicidad}

La necesidad de atraer al lector desde las redes sociales hasta la web a golpe de titular para generar beneficios va a propiciar un entorno donde los titulares más llamativos o sensacionalistas se verán recompensados. Las principales páginas españolas de contenido sesgado o directamente falsos que obtienen beneficios con este modelo de publicidad de Google, la Google Display Network, son Periodista Digital, OK Diario, Esdiario, Gaceta, Caso Aislado, La Tribuna de Cartagena, Mediterráneo Digital y Digital Sevilla (Peinado y Muela, 2018).

Por otro lado, los anunciantes tienen poder suficiente para influir en los contenidos de los medios, pero no parece que hasta el momento les haya incomodado ninguno de los contenidos generados por estas webs. Hasta ahora el único caso conocido ha sido la retirada de publicidad de Nissan y Toyota tras una campaña de presión debida a un contenido machista en Mediterráneo Digital (Llanos, y Rodríguez-Pina, 2017).

Muchas empresas rechazan patrocinar a sus enemigos ideológicos o a aquellos que puedan dañar su reputación y, sin embargo, excepto el caso anteriormente mencionado, no parece que los contenidos de estas webs, y de Caso Aislado en concreto, estén provocando incomodidad en las empresas anunciantes, que no ha castigado estos contenidos por su falta de rigor o su tendencia al extremismo del espectro ideológico (Peinado y Muela, 2018).

\subsection{Tercer filtro: ¿quién necesita fuentes?}

En los medios convencionales, la imposibilidad de tener redactores y corresponsales en todo el mundo hace a los medios depender de otras fuentes de generación de noticias: agencias, instituciones, gabinetes de prensa... Con tan sólo cinco redactores en plantilla, es de suponer que Caso Aislado sobrevive directamente reelaborando noticias generadas por agencias y otros medios de comunicación.

Las fuentes de información con poder pueden permitirse vetar el acceso a medios críticos, como en el caso de Vox con La Sexta justo cuando su progresiva visibilidad en redes y encuestas lo catapulta a la agenda informativa. Eso refuerza su postura de outsider del sistema. Como vimos en el estudio sobre su presencia en la campaña 
electoral, pueden permitirse esta posición porque su visibilidad en la agenda no se pone en peligro con este veto.

Sería interesante analizar, sin embargo, como el ecosistema que apenas hemos esbozado de estas webs de ultraderecha dentro de Internet y las redes sociales, se convierte en una red de fuentes de información en sí mismas donde las fuentes de la noticia no cumplen la función de aportar veracidad sino que en una suerte de inversión, no se busca la presunción de objetividad sino todo lo contrario, las noticias se mueven dentro de un entorno que busca ver reafirmada sus posturas ideológicas, el sesgo de confirmación.

\subsection{Cuarto filtro: reforzadores de opinión. The Movement}

Este ecosistema de webs conectadas puede ejercen también una función de reforzador de opinión. Además, colocan a menudo como "enemigos" a periodistas críticos con VOX, englobándolos en una serie de ataques contra la formación que aparece como víctima.

Pero además, el ‘equipo’ europeo de Bannon cuenta también con su propio think tank, fundado por Mischaël Modrikamen, el líder del partido conservador de Bélgica, Parti Populaire y que tiene como objetivo "promover el respeto al Estado de derecho, la libre empresa, la soberanía de naciones, la efectividad de las fronteras nacionales, la consulta popular, la lucha contra el Islam radical, un enfoque científico y no dogmático de los fenómenos climáticos y la defensa de Israel como un estado soberano en su territorio histórico" (The Movement, 2018).

Según sus estatutos, The Movement es "el vínculo entre el movimiento iniciado por el presidente D.J. Trump en EEUU y ciudadanos y movimientos políticos en otros países, incluidos los actores de la campaña para el Brexit, y quienes se reconocen en los principios fundacionales definidos anteriormente. La entidad tiene como objetivo reunir el apoyo por todos los medios adecuados, incluidos los financieros, coordinar y reforzar mutuamente la acción de estos ciudadanos y movimientos".

El propio Steve Bannon aseguró que su intención al promover la creación de la fundación era liderar una revuelta de la ultraderecha en Europa con el objetivo puesto en las elecciones europeas de esta primavera, funcionando como un think tank que genere estudios, análisis de datos y asesoría en comunicación (Hines, 2018) por lo que parece no caber duda de la intención de una estrategia comunicativa coordinada por parte de la ultraderecha global.

\subsection{Quinto filtro: convergencia en la ideología dominante}

Aunque el anticomunismo no es uno de los ejes fundamentales del discurso de Caso Aislado, si se realiza una búsqueda en su web, se puede observar que usan a menudo de modo descalificativo expresiones como "progre", presente en 4 titulares de su web del periodo analizado con connotaciones negativas y uno con la expresión 'comunista' usada como descalificativo.

Sin embargo, desde una aproximación de la alineación con la ideología dominante y desde el uso que hace la ultraderecha de la construcción social del enemigo, la concepción de este se amplía, como vimos en al análisis de contenido y se incluyen a las feministas, el independentismo catalán, y los inmigrantes. Estos temas y el enfoque desde el que se los trata están presentes en los medios de comunicación 
de masas, por lo que es posible entender que el discurso se alinea con la ideología dominante.

\section{7. ¿Fuera de la agenda? Conclusiones y cuestiones para el debate}

La hipótesis de partida es que el uso de los portales de fake news y su viralidad no son usados como una alternativa a los medios de comunicación tradicionales, ya que el acceso a estos no se encuentra vedado, sino como una herramienta complementaria con un papel bien definido.

¿Qué papel juegan las webs de fake news, por tanto, en este panorama? Si se lee el poder como hegemonía se puede entender que la producción de consentimiento es un proceso descentralizado que se produce a través de dispositivos difusos (Sierra y Vázquez, 2006). Los portales del entorno de la ultraderecha, como Caso Aislado, son una pieza más de un dispositivo que impone sus mensajes por acumulación a través de diferentes medios. Las webs de fake news refuerzan y se retroalimentan de un mensaje neoconservador que no se ve marginado en los medios de comunicación. Cabría preguntarse si son usadas como punta de lanza para mover los marcos discursivos progresivamente hacia posiciones de derecha más extremas. Es posible apuntar el esbozo de una red de formaciones de extrema derecha entre las que existe una coordinación internacional para compartir recursos y estrategias comunicativas, siendo una de sus principales herramientas este ecosistema de webs dominados por las noticias falsas.

En el aspecto teórico y aplicando el modelo de propaganda revisado de Chomsky y Herman, se puede comprobar que ninguno de los filtros determinados supone un obstáculo para la incorporación a la agenda del discurso de la ultraderecha. Algunos estudios realizados en otros países han establecido la influencia de las webs de fake news en la agenda de los medios tradicionales en ciertos temas y actores políticos (Guo y Vargo, 2018). Aunque es esencial realizar dicha investigación en profundidad comprobando la presencia de estos ejes temáticos en una muestra representativa de los medios de comunicación de masas más relevantes, sólo es posible por ahora rastrear su presencia a través de estudios ya realizados para intentar apuntar en una primera instancia nuestra hipótesis de la cabida del discurso de la ultraderecha en el discurso hegemónico.

En el caso del discurso contra la inmigración parece que la ultraderecha no ha descubierto un ítem discursivo nuevo, como ya analizaron Van Dijk y otros (Dijk y Bastida, 2006; Martínez Lirola, 2008), los delitos cometidos por inmigrantes son un eje fundamental en el discurso de los medios de comunicación. Todas las falsas noticias sobre presuntos delitos cometidos por inmigrantes coinciden y refuerzan la línea del discurso dominante en los medios que genera alarma social frente a la inmigración y una polarización entre nosotros y ellos.

El auge de un discurso neomachista y hostil al feminismo ha sido analizado en las industrias culturales, observándose un discurso que defiende una supuesta igualdad frente a las exigencias exageradas de un feminismo radical y que "aparecen, igual que sucedió en los años sesenta del siglo XX y luego en los noventa, como respuesta ante los avances en los derechos de las mujeres. No porque se haya conseguido la igualdad -así lo explicaba Susan Faludi en 1991- sino porque parecía probable que estuviera cerca” (Menéndez Menéndez, 2017). 
Se ha establecido el posicionamiento de Vox como víctima de los medios de comunicación de masas, al tratarse de uno de los ejes temáticos principales de las noticias donde se hacía referencia a ellos. Este posicionamiento de Vox y de los portales digitales de fake news como outsiders e incómodos para el discurso dominante, queda refutado con el estudio que analiza la presencia de Vox en los medios de comunicación, analizando la cobertura realizada por El País y El Mundo desde el nacimiento del partido (2013) con especial incidencia en el periodo de las elecciones andaluzas (Olalla et al., 2019)

Con estos datos, es posible afirmar que la pretensión de la ultraderecha de su exclusión por parte de los medios de comunicación tradicionales no se ajusta a lo observado y se puede al menos esbozar la hipótesis de que, en realidad, sus ejes discursivos si tienen cabida en el marco del discurso hegemónico.

El argumento por parte de la ultraderecha, concretamente en el caso de Vox, de que el uso de los portales de noticias y la difusión de redes sociales es debido a su marginación de los medios de comunicación tradicionales no se corresponde, por tanto, con la realidad. No sólo partiendo del modelo de propaganda comprobamos que no hay elementos teóricos que impidan que su discurso se quede fuera de la agenda mediática, sino que, además, a través de algunos ejemplos podemos plantearnos que sus ejes temáticos están representados e imbricados en el discurso dominante. Pese a la pretendida figura de outsiders, su capacidad de colocar sus temas en la agenda nos hace plantearnos que sus contenidos se alinean con los intereses de la ideología dominante y usan, por tanto, esta figura para obtener réditos políticos ante la desafección ciudadana por la política tradicional.

\section{Referencias}

Abascal, S. (2018, 19 septiembre). Los valores de VOX. Visioncoach. [post]. Recuperado de: https://visioncoach.es/2018/09/19/los-valores-de-vox-por-santiago-abascal/]

Allcott, H., y Gentzkow, M. (2017). Social Media and Fake News in the 2016 Election. Journal Of Economic Perspectives, 31(2), 211-236. doi: 10.1257/jep.31.2.211

Andreu, J. (2008). Establecimiento de la Agenda: Un Análisis Longitudinal sobre la influencia de la prensa en los barómetros del CIS. Metodología de Encuestas, 10, 93-118

Andreu, J. (2012) La descodificación de la agenda: Un modelo analítico para el conocimiento manifiesto y latente de la agenda pública. Intangible Capital 8(3), 520-547. doi: 10.3926/ ic. 380

Bovet, A. y Makse, H. (2019). Influence of fake news in Twitter during the 2016 US presidential election. Nature Communications, 10 (7). doi: 10.1038/s41467-018-07761-2

Boyd-Barrett, O. (2004). Judith Miller, The New York Times, and the propaganda model. Journalism Studies, 5(4), 43-449. doi: 10.1080/14616700412331296383

Cadwalladr, C., y Graham-Harrison, E. (2018, 17 de marzo). Revealed: 50 million Facebook profiles harvested for Cambridge Analytica in major data breach. The Guardian [en línea]. Recuperado de: https://www.theguardian.com/news/2018/mar/17/cambridge-analyticafacebook-influence-us-election

Campos Mello, P. (2018, 18 de ocutbre). Empresários bancam campanha contra o PT pelo WhatsApp. Fohla De S.Paulo [en línea]. Recuperado de: https://www1.folha.uol.com.br/ poder/2018/10/empresarios-bancam-campanha-contra-o-pt-pelo-whatsapp.shtml 
Camiñas Hernández, T. (2003). Los medios de comunicación como soporte de la propaganda: el caso del 11-s en la prensa diaria española de referencia, a través de los expertos. Tesis doctoral. Universidad de Málaga. Recuperado de: https://dialnet.unirioja.es/servlet/ tesis? codigo $=217038$

Chomsky, N., y Herman, E. (2009). Los guardianes de la libertad: propaganda, desinformación y consenso en los medios de comunicación de masas (6 ed). Barcelona. Crítica.

Chomsky, N. (2007). Ilusiones necesarias: control del pensamiento en las sociedades democráticas. La Plata. Terramar Ediciones.

Centro de Investigaciones Sociológicas (2017). Barómetro del CIS. Recuperado de: http:// datos.cis.es/pdf/Es3191mar_A.pdf

Centro de Investigaciones Sociológicas (2018). Barómetro del CIS. Recuperado de: http:// datos.cis.es/pdf/Es3234mar_A.pdf

Dijk, T. y Bastida, M. (2006). Me $\overline{d i o s}$ de comunicación e inmigración. Madrid. CAM, Obra Social.

European Research Council (2018). Selective Exposure to Misinformation: Evidence from the consumption of fake news during the 2016 U.S. presidential campaign. Recuperado de: http://www.dartmouth.edu/ nyhan/fake-news-2016.pdf

European Commission (2019) Factsheet on Tackling online disinformation. Digital Single Market. Recuperado de: https://ec.europa.eu/digital-single-market/en/news/factsheettackling-online-disinformation

European Commission (2019). Fake news and online disinformation. Digital Single Market. Recuperado de: https://ec.europa.eu/digital-single-market/en/fake-news-disinformation

Llanos, H. y Rodríguez-Pina, G., (2017, 26 de enero). Una tuitera logra que varias marcas quiten su publicidad de un medio online por machismo. Verne, El País [en línea]. Recuperado de:https://verne.elpais.com/verne/2017/01/26/articulo/1485444899_460706. html

Gómez Masjuán, M.E. (2010). La construcción mediática de Cuba. Un análisis del discurso periodístico del The Washington Post. Revista Latina de Comunicación Social. Recuperado de: http://www.redalyc.org/articulo.oa?id=81915723008

Guo, L., y Vargo, C. (2018). "Fake News" and Emerging Online Media Ecosystem: An Integrated Intermedia Agenda-Setting Analysis of the 2016 U.S. Presidential Election. Communication Research. doi: 10.1177/0093650218777177

Hall, S. (2018). El largo camino de la renovación. El thatcherismo y la crisis de la izquierda. Madrid. Lengua de Trapo.

Herman, E. \& McChesney, R. (1999). Los medios globales: los nuevos misioneros del capitalismo corporativo. Madrid. Cátedra.

Hines, N. (2018, 20 de julio). Inside Bannon's Plan to Hijack Europe for the Far-Right. The Daily Beast. Recuperado de: https:/www.thedailybeast.com/inside-bannons-plan-tohijack-europe-for-the-far-right

Junquera, N. (2018, 5 de diciembre). Qué tienen en común Vox, el jefe de campaña de Trump y Le Pen. El País [en línea]. Recuperado de: https://elpais.com/politica/2018/12/04/ actualidad/1543949909_697562.html

Klinger, U., y Svensson, J. (2014). The emergence of network media logic in political communication: A theoretical approach. New Media \& Society, 17(8), 1241-1257. doi: $10.1177 / 1461444814522952$

Knight Foundation (2018). Disinformation, 'Fake News' and Influence Campaigns on Twitter. Recuperado de: https://knightfoundation.org/reports/disinformation-fake-news-andinfluence-campaigns-on-twitter 
Jiménez Losantos, F. (2018, 9 de diciembre). VOX, prueba de vida de la democracia española. Libertad Digital. Recuperado de: https://www.libertaddigital.com/opinion/federicojimenez-losantos/vox-prueba-de-vida-de-la-democracia-espanola-86672/

Mazzoleni, G., y Bracciale, R. (2018). Socially mediated populism: the communicative strategies of political leaders on Facebook. Palgrave Communications, 4(1). doi: 10.1057/ s41599-018-0104-X

Menéndez Menéndez, M.I. (2017) Entre Neomachismo y retrosexismo: antifeminismo en industrias culturales. Prisma Social. Recuperado de: http://www.redalyc.org/articulo. oa? id=353752825001

McChesney, R. (2008). The political economy of media: enduring issues, emerging dilemmas. New York. Monthly Review Press.

McChesney, R. (2013). Desconexión digital : cómo el capitalismo está poniendo a Internet en contra de la democracia. Barcelona.El Viejo Topo.

Negre, J. (2018, 9 de diciembre). El joven propagandista de Vox que montó a Abascal en un caballo. El Mundo. Recuperado de: https:/www.elmundo.es/cronica/2018/12/09/5c0a9d 12fdddff0e388b4606.html

Olalla, S., Chueca, E., y Padilla, J. (2019, 11 de enero). Spain is no longer exceptional: Mainstream media and the far-right party Vox. Recuperado de: http://blogs.lse.ac.uk/ eurocrisispress/2019/01/10/spain-is-no-longer-exceptional-mainstream-media-and-thefar-right-party-vox/

Pedro, Joan (2009): Evaluación crítica del Modelo de Propaganda de Herman y Chomsky. RLCS, Revista Latina de Comunicación Social, 64, 210-227. doi: 10.4185/RLCS-642009-818-210-227

Peinado, F., y Muela, D. (2018, 23 de mayo). El negocio de la manipulación digital en España. El País [en línea]. Recuperado de: https://elpais.com/politica/2018/05/17/ actualidad/1526571491_535772.html

Pérez-Curiel, C. y Limón Naharro, P. (2019). Political influencers. A study of Donald Trump's personal brand on Twitter and its impact on the media and users. Communication \& Society 32(1), 57-75.

Pineda, A. y Romero, L. (2011). Estructura mediática y neoconservadurismo: Rupert Murdoch y su desembarco en EE.UU. Estudios sobre el mensaje periodístico. Vol. 17(1), 195-214. Madrid, Servicio de Publicaciones de la Universidad Complutense.

Pineda, A. (2009). Algunas implicaciones de la teoría de la Economía Política de la Comunicación para la investigación de la propaganda., RLCS, Revista Latina de Comunicación Social, 64.553-562. doi: 10.4185/RLCS-64-2009-844-553-562

Rachman, A. y Yunianti, S., 2017. Critical Discourse Analysis in Donald Trump presidential campaign to win American's heart. TELL Journal. Vol.5, núm, 2. Recuperado de: http:// journal.um-surabaya.ac.id/index.php/Tell/article/download/388/543

Ramírez, V., y Castellón, J. (2018, 9 de octubre). 'Caso Aislado', el fabricante español de 'fake news' vinculado a VOX. La Sexta. Recuperado de: https://www.lasexta.com/ noticias/nacional/asi-trabajan-caso-aislado-fabricante-espanol-fake-news-vinculado-vox 201810095bbcb81e0cf22dcc97718ef6.html

Rieder, B. (2013). Studying Facebook via data extraction. Proceedings of the 3rd Annual ACM Web Science Conference, WebSci. 346-355. doi: 10.1145/2464464.2464475.

Rodríguez Andrés, R. (2016). El ascenso de los candidatos outsiders como consecuencia de las nuevas formas de Comunicación Política y la desafección ciudadana. Comunicación y Hombre, 12, 73-95. Recuperado de: https://www.redalyc.org/articulo.oa?id=129446703005 
Tandoc, E., Wei Lim, Z. y Ling, R. (2018) Defining "Fake News", Digital Journalism, Vol. 6(2),137-153. doi: 10.1080/21670811.2017.1360143

The Movement (2018). Estatutos de The Movement. Recuperado de: https://es.scribd.com/ document/395205578/Estatutos-The-Movement

Vázquez Liñán, M., y Sierra Caballero, F. (2006). La construcción del consenso. Revisitando el modelo de propaganda de Noam Chomsky y Edward S. Herman. Madrid. Siranda.

Vlatković, S. (2018). New Communication Forms and Political Framing: Twitter in Donald Trump's Presidential Campaign. AM Journal of Art and Media Studies, (16), p.123. doi: 10.25038/am.v0i16.259 